
\title{
$\begin{array}{ll}\text { Research Square } & \begin{array}{l}\text { Preprints are preliminary reports that have not undergone peer review. } \\ \text { They should not be considered conclusive, used to inform clinical practice, } \\ \text { or referenced by the media as validated information. }\end{array}\end{array}$
}

\section{Nationwide Study of Mortality After Abdominal Aortic Aneurysm Repair in France 2010- 2019}

Juliette Raffort ( $\square$ juliette.raffort@hotmail.fr)

Université Côte d’Azur, CHU, Inserm U1065, C3M, Nice, France

\section{Fabien Lareyre}

Department of Vascular Surgery, Hospital of Antibes Juan-les-Pins, France

\section{Roxane Fabre}

CoBTeK lab, Université Côte d’Azur, Nice, France

\section{Ziad Mallat}

Division of Cardiovascular Medicine, University of Cambridge, Cambridge, UK

\section{Christian Pradier}

Public Health Department, University Hospital of Nice, Université Côte d'Azur, Nice, France

\section{Laurent Bailly}

Public Health Department, University Hospital of Nice, Université Côte d’Azur, Nice, France

\section{Research Article}

Keywords: Nationwide Study of Mortality, Aortic Aneurysm, cardio-metabolic comorbidities

Posted Date: February 5th, 2021

DOI: https://doi.org/10.21203/rs.3.rs-174111/v1

License: (c) (i) This work is licensed under a Creative Commons Attribution 4.0 International License. Read Full License 


\section{Abstract}

Objective

The aim of this nationwide study was to analyze mortality in patients undergoing AAA repair and to explore the association between cardio-metabolic factors with clinical presentation and post-operative mortality.

Methods

This ten-year retrospective, multicenter study based on the French National electronic health data included patients undergoing AAA repair between 2010 and 2019. Post-operative mortality was analyzed using Kaplan-Meier curve survival and Log-Rank tests. A multivariate regression analysis was performed to calculate Hazard Ratios.

Results

Over 79935 patients who underwent AAA repair, 61146 patients (76.5\%) had at least one hospital-readmission after the AAA repair, for a mean follow-up of 3.5 years +/- 2.5. The in-hospital mortality was $4581(5.8 \%)$ and the total postoperative mortality was 12400 (20.3\%). Age over 64 years old, the presence of AAA rupture and in-hospital readmission at 30-day were predictors of post-operative mortality $(\mathrm{HR}=1.59 \mathrm{Cl} 95 \%=1.51-1.67 ; \mathrm{HR}=1.49 \mathrm{Cl} 95 \%=1.36-1.62$ and $\mathrm{HR}=1.92$, $\mathrm{Cl} 95 \%=1.84-2.00)$. Cardio-metabolic factors were negatively associated with AAA rupture $(\mathrm{OR}=0.44 \mathrm{CI} 95 \%=0.41-0.47$ for dyslipidemia, $\mathrm{OR}=0.75 \mathrm{Cl} 95 \%=0.70-0.8$ for hypertension).

Conclusion

The negative association observed between cardio-metabolic comorbidities and AAA rupture suggests that AAA may be underdiagnosed in patients without known comorbidities and highlights the interest of AAA screening.

\section{Introduction}

Abdominal aortic aneurysm (AAA) corresponds to a focal dilatation of the aorta and is associated with high rate of mortality in case of aortic rupture ${ }^{1-3}$. The treatment of AAA relies on surgical approaches and can be performed using open repair or endovascular aneurysm repair (EVAR) ${ }^{1,3}$. The indication for AAA surgical repair depends on the balance between the risk of aneurysmal growth and rupture, and the operative risk.

Cardiovascular risk factors and metabolic disorders play a key role in AAA development and they can also impact the postoperative outcomes of patients ${ }^{1,3-5}$. Cardiovascular events have been identified as one of the leading cause of death in patients undergoing AAA repair ${ }^{1,6}$. Screening for cardiovascular and metabolic risk factors in the general population varies between countries and resources and most guidelines recommend a mixture of opportunistic and systematic screening ${ }^{7}$. The European Society for vascular Surgery (ESVS) guidelines currently recommend a systematic screening of cardiovascular risk factors in patients undergoing AAA repair in order to assess and limit the operative risks ${ }^{1}$. While several retrospective nationwide studies investigating the outcomes of patients undergoing AAA repair have been performed in European countries ${ }^{6,8,9}$, it has not yet been performed in France. Such approach is of interest to evaluate the clinical characteristics and comorbidities of patients operated for an AAA and provide a state of surgical practice.

In this 10-year descriptive nationwide study, we aimed to analyze mortality in patients undergoing AAA repair in France and to explore the association between cardio-metabolic factors, AAA clinical presentation and post-operative mortality.

\section{Methods}

\section{Study design and data collection}

Page 2/16 
This population-based, multicenter, cohort study was based on the French National Health insurance information system and was conducted in accordance with the French National Health Data Institute regulation. The protocol was approved by the Institutional Review Board of the University Hospital of Nice and by the French National Health Information System (Système National des Données de Santé, SNDS). This electronic database has been previously described ${ }^{10,11}$ and informed consent was waived for this type of study in accordance with the French National Health Data Institute regulation. Information was extracted from anonymous discharge reports completed and coded at the end of each hospital stay.

Eligible patients where those undergoing an AAA repair for the first time in any public or private hospital in France between 2010 and 2019. AAA repair was classified into open repair or EVAR according to the Common Classification of Medical Acts (CCAM). Collected data included the general characteristics of patients, the comorbidities, the type of AAA repair, the presence of an aneurysm rupture or not, and the post-operative outcomes (mortality and in-hospital readmission at 30 days). Comorbidities were defined according to the International Classification of Diseases (ICD-10). The codes used are presented in the Supplementary data.

The all-cause mortality was analyzed based on the French National electronic health data that allowed to identify all deaths registered during an ulterior hospital stay over a ten-year period. In-hospital mortality was defined as deaths occurring during the first hospital stay for AAA repair. One-year mortality corresponded to the number of deaths occurring during the year following the first AAA repair. Total mortality was defined as the number of deaths recorded from the first AAA repair until the last follow-up. In-hospital readmission at 30 days included all re-hospitalization occurring within 30 days after discharge from the first AAA repair whatever the cause.

\section{Statistical analysis}

Results for quantitative variables were expressed as means +/- standard deviation (SD) and as percentages for categorical variables. Group differences were analyzed using Student t-test for quantitative variables and Chi-2 test for qualitative variables.

The factors associated with AAA rupture were searched using a logistic regression including the general characteristics of patients (age, sex) and the comorbidities. Statistically significant results were presented using odds ratio with $95 \%$ confidence interval $(95 \% \mathrm{Cl})$.

A survival analysis was performed in order to identify risk factors associated with post-operative mortality. Only patients who had at least one follow-up at hospital after the first AAA repair recorded in the National French electronic health data between 2010 and 2019 were included. The release date from the hospital after the first AAA repair was considered as the starting point and the follow-up was calculated from this date until the last hospital stay. The analysis excluded deaths that occurred during the hospital stay for the first AAA repair. Kaplan-Meier curve survival and Log-Rank tests were used to analyze the risk of post-operative mortality. Multivariate regression analysis using a Cox proportional model was performed to calculate Hazard Ratios with $95 \% \mathrm{Cl}$. The hypothesis of the proportional risk was evaluated according to the survival curves using the double negative logarithm function. The factors which did not comply with the hypothesis of the proportional risk were excluded from the model. A two-tailed P-value $<0.05$ was considered as statistically significant. Statistical analysis was performed using SAS Enterprise 5.1 (SAS Institute, Cary, NC).

\section{Results}

\section{Characteristics of patients who underwent AAA repair in France between 2010 and 2019}


Between 2010 and 2019, 79935 patients underwent AAA repair in France and were included in this study; 31041 (38.8\%) had an open repair while 48894 (61.2\%) underwent EVAR. The flow chart of the study population is depicted in Fig. 1. The mean age of the population was $71.6+/-9.7$ years and $90.6 \%$ were men (Table 1 ). The in-hospital mortality during the first AAA repair was 4581 (5.8\%). The mean follow-up was $2.7+/-2.7$ years. The 1-year mortality was 7467 (9.3\%) and the total mortality over the 10-year study was 16986 (21.3\%). 
Table 1

Characteristics of patients operated for an abdominal aortic aneurysm classified according to the type of surgery and the presence of a rupture

\begin{tabular}{|c|c|c|c|c|c|c|c|}
\hline & $\begin{array}{l}\text { Total } \\
\mathrm{N}=79935\end{array}$ & $\begin{array}{l}\text { Open } \\
\text { repair } \\
N=31041\end{array}$ & $\begin{array}{l}\text { EVAR } \\
N=48894\end{array}$ & p & $\begin{array}{l}\text { Non ruptured } \\
\text { AAA } \\
\text { N= } 75257\end{array}$ & $\begin{array}{l}\text { Ruptured } \\
\text { AAA } \\
\mathrm{N}=4678\end{array}$ & p \\
\hline $\mathrm{Age}^{\mathrm{y}}$ & $71.6 \pm 9.7$ & $68.7 \pm 9.3$ & $73.4 \pm 9.1$ & $\begin{array}{l}< \\
0.001\end{array}$ & $71.6 \pm 9.7$ & $72.1 \pm 10.4$ & $\begin{array}{l}<.001 \\
0.001\end{array}$ \\
\hline Sex ratio M/F (\%) & $90.6 / 9.4$ & $90.8 / 9.2$ & $90.4 / 9.6$ & 0.09 & $90.7 / 9.3$ & $88.8 / 11.2$ & $\begin{array}{l}<.001 \\
0.001\end{array}$ \\
\hline In-hospital stay & $10.0 \pm 11.1$ & $13.9 \pm 13.9$ & $7.5 \pm 7.9$ & $<0.001$ & $9.7 \pm 10.2$ & $15.5 \pm 20.0$ & $\begin{array}{l}<.001 \\
0.001\end{array}$ \\
\hline Mean follow-upy & $2.7 \pm 2.7$ & $2.8 \pm 2.8$ & $2.7 \pm 2.5$ & $<.001$ & $2.8 \pm 2.7$ & $1.6 \pm 2.5$ & $\begin{array}{l}<.001 \\
0.001\end{array}$ \\
\hline Rupture & $4678(5.9)$ & $\begin{array}{l}4531 \\
(14.6)\end{array}$ & $147(0.3)$ & $<001$ & & & \\
\hline Total mortality & $\begin{array}{l}16986 \\
(21.3)\end{array}$ & $\begin{array}{l}7143 \\
(23.0)\end{array}$ & $\begin{array}{l}9843 \\
(20.1)\end{array}$ & $<0.001$ & 14561 (19.4) & $\begin{array}{l}2425 \\
(51.8)\end{array}$ & $\begin{array}{l}< \\
0.001\end{array}$ \\
\hline In-hospital mortality & $4581(5.8)$ & $\begin{array}{l}3324 \\
(10.7)\end{array}$ & $1257(2.6)$ & $<0.001$ & 2708 (3.6) & $\begin{array}{l}1873 \\
(40.0)\end{array}$ & $\begin{array}{l}<.001 \\
0.001\end{array}$ \\
\hline 1-year mortality & 7467 (9.3) & $\begin{array}{l}4129 \\
(13.1)\end{array}$ & $3338(6.8)$ & $<001$ & $5419(7.2)$ & $\begin{array}{l}2048 \\
(43.8)\end{array}$ & $\begin{array}{l}< \\
0.001\end{array}$ \\
\hline Arterial hypertension & $\begin{array}{l}60897 \\
(76.2)\end{array}$ & $\begin{array}{l}23283 \\
(75.0)\end{array}$ & $\begin{array}{l}37614 \\
(76.9)\end{array}$ & $<.001$ & 57853 (76.9) & $\begin{array}{l}3044 \\
(65.1)\end{array}$ & $\begin{array}{l}<.001 \\
0.001\end{array}$ \\
\hline Dyslipidemia & $\begin{array}{l}40989 \\
(51.3)\end{array}$ & $\begin{array}{l}15895 \\
(51.2)\end{array}$ & $\begin{array}{l}25094 \\
(51.3)\end{array}$ & 0.75 & 39504 (52.5) & $\begin{array}{l}1485 \\
(31.7)\end{array}$ & $\begin{array}{l}< \\
0.001\end{array}$ \\
\hline T2D & $\begin{array}{l}16408 \\
(20.5)\end{array}$ & $\begin{array}{l}5931 \\
(19.1)\end{array}$ & $\begin{array}{l}10477 \\
(21.4)\end{array}$ & $<.001$ & 15718 (20.9) & $690(14.8)$ & $\begin{array}{l}<.001 \\
0.001\end{array}$ \\
\hline T1D & 3092 (3.9) & 1049 (3.4) & $2043(4.2)$ & $<001$ & $2973(4.0)$ & $119(2.5)$ & $\begin{array}{l}<.001 \\
0.001\end{array}$ \\
\hline Smoking & $\begin{array}{l}19225 \\
(24.1)\end{array}$ & $\begin{array}{l}8964 \\
(28.9)\end{array}$ & $\begin{array}{l}10261 \\
(21.0)\end{array}$ & $<001$ & $18238(24.2)$ & $987(21.1)$ & $\begin{array}{l}< \\
0.001\end{array}$ \\
\hline Obesity & $\begin{array}{l}17541 \\
(21.9)\end{array}$ & $\begin{array}{l}6352 \\
(20.5)\end{array}$ & $\begin{array}{l}11189 \\
(22.9)\end{array}$ & $\begin{array}{l}< \\
0.001\end{array}$ & $16738(22.2)$ & $803(17.2)$ & $\begin{array}{l}<.001 \\
0.001\end{array}$ \\
\hline SAOS & $\begin{array}{l}8403 \\
(10.5)\end{array}$ & 2769 (8.9) & $\begin{array}{l}5634 \\
(11.5)\end{array}$ & $\begin{array}{l}< \\
0.001\end{array}$ & 8105 (10.8) & $298(6.4)$ & $\begin{array}{l}< \\
0.001\end{array}$ \\
\hline CHF & $\begin{array}{l}28499 \\
(35.7)\end{array}$ & $\begin{array}{l}10170 \\
(32.8)\end{array}$ & $\begin{array}{l}18329 \\
(37.5)\end{array}$ & $\begin{array}{l}< \\
0.001\end{array}$ & 27138 (36.1) & $\begin{array}{l}1361 \\
(29.1)\end{array}$ & $\begin{array}{l}< \\
0.001\end{array}$ \\
\hline $\begin{array}{l}\text { Chronic respiratory } \\
\text { disease }\end{array}$ & $\begin{array}{l}23096 \\
(28.9)\end{array}$ & $\begin{array}{l}9075 \\
(29.2)\end{array}$ & $\begin{array}{l}14021 \\
(28.7)\end{array}$ & 0.09 & 22051 (29.3) & $\begin{array}{l}1045 \\
(22.3)\end{array}$ & $\begin{array}{l}< \\
0.001\end{array}$ \\
\hline Chronic kidney disease & $\begin{array}{l}16332 \\
(20.4)\end{array}$ & $\begin{array}{l}5561 \\
(17.2)\end{array}$ & $\begin{array}{l}10771 \\
(22.0)\end{array}$ & $\begin{array}{l}< \\
0.001\end{array}$ & $15487(20.6)$ & $845(18.1)$ & $\begin{array}{l}< \\
0.001\end{array}$ \\
\hline Stroke & $\begin{array}{l}14959 \\
(18.7)\end{array}$ & $2394(7.7)$ & $4403(9.0)$ & $\begin{array}{l}< \\
0.001\end{array}$ & 14337 (19.1) & $622(13.3)$ & $\begin{array}{l}< \\
0.001\end{array}$ \\
\hline
\end{tabular}




\begin{tabular}{|c|c|c|c|c|c|c|c|}
\hline & $\begin{array}{l}\text { Total } \\
\mathrm{N}=79935\end{array}$ & $\begin{array}{l}\text { Open } \\
\text { repair } \\
N=31041\end{array}$ & $\begin{array}{l}\text { EVAR } \\
N=48894\end{array}$ & $\mathbf{p}$ & $\begin{array}{l}\text { Non ruptured } \\
\text { AAA } \\
\text { N=75257 }\end{array}$ & $\begin{array}{l}\text { Ruptured } \\
\text { AAA } \\
\text { N=4678 }\end{array}$ & $\mathbf{p}$ \\
\hline \multicolumn{8}{|c|}{ AAA: abdominal aortic aneurysm } \\
\hline \multicolumn{8}{|c|}{ CHF: congestive heart failure } \\
\hline \multicolumn{8}{|l|}{ d: days } \\
\hline \multicolumn{8}{|c|}{ EVAR: endovascular aneurysm repair } \\
\hline \multicolumn{8}{|c|}{ F: female } \\
\hline \multicolumn{8}{|l|}{ M: male } \\
\hline \multicolumn{8}{|c|}{ SAOS: Sleeping Apnea Obstructive Syndrome } \\
\hline \multicolumn{8}{|c|}{ T1D: type 1 diabetes } \\
\hline \multicolumn{8}{|c|}{ T2D: type 2 diabetes } \\
\hline$y$ : years & & & & & & & \\
\hline
\end{tabular}

In $94.1 \%$ of cases, the AAA was not ruptured $(n=75257)$, while ruptured aneurysms concerned less than $6 \%$ of patients ( $n$ $=4678 ; 5.9 \%$ ). Most of the patients with ruptured AAA were treated using open repair (4 531/4678,96.8\%). The total mortality was significantly higher in patients with AAA rupture (2 425,51.8\%) compared to those without rupture (14 561, $19.4 \%, P<0.001)$. The in-hospital mortality was significantly higher in patients who had open repair compared to those who underwent EVAR $(10.7 \%$ vs $2.6 \%, \mathrm{P}<0.001)$. In case of AAA rupture, the death occurred more frequently during the hospital stay of the first AAA repair (1 873 over $2425,77.2 \%$ ) while it represented only $18.7 \%$ (2 708 over 14561 ) of the total deaths in patients without rupture.

The prevalence of comorbidities was significantly lower in patients with AAA rupture compared to those without rupture. This included metabolic syndrome related disorders such as T2D (14.8\% vs $20.9 \%, \mathrm{P}<0.001)$, arterial hypertension $(65.1 \%$, vs $76.9 \%, \mathrm{P}<0.001)$, dyslipidemia $(31.7 \%$ vs $52.5 \%, \mathrm{P}<0.001)$, obesity $(17.2 \%$ vs $22.2 \%, \mathrm{P}<0.001)$ or SAOS $(6.4 \%$ vs $10.8 \%$, $\mathrm{P}<0.001)$. The prevalence of cardiovascular risk factors and diseases was also lower, with a lower rate of smoking $(21.1 \%$ vs $24.2 \%, \mathrm{P}<0.001)$, CHF $(29.1 \%$ vs $36.1 \%, \mathrm{P}<0.001)$ or past history of stroke $(13.3 \%$ vs $19.1 \%, \mathrm{P}<0.001)$.

\section{Association of cardio-metabolic factors with post-operative mortality of patients who underwent AAA repair}

The prevalence of AAA rupture and the post-operative outcomes of patients were analyzed according to the presence or not of cardio-metabolic comorbidities (Table 2). As previously observed, the prevalence of AAA rupture was significantly lower in patients with cardio-metabolic diseases including dyslipidemia, arterial hypertension, diabetes, obesity, SAOS, smoking, CHF or stroke. Similarly, the in-hospital mortality was significantly lower in patients presenting these cardio-metabolic comorbidities compared to those who did not. The same trend was observed for the 1-year mortality, except for the history of CHF and stroke. Indeed, the 1-year mortality was higher in patients with CHF $(2855,10.0 \%$ vs $4612(9.0 \%), P<0.01)$ or patients with stroke $(702,10.3 \%$ vs $6765,9.2 \%, \mathrm{P}<0.01)$ compared to those who did not have these comorbidities. The associations between cardio-metabolic diseases and the total mortality were more heterogeneous. The total mortality was significantly lower in patients presenting dyslipidemia, obesity, SAOS or smoking compared to those who did not have these comorbidities. Note that smokers were significantly younger than the non-smoking group (66.6 years vs 73.2 years, $\mathrm{P}$ $<0.01)$. On the opposite, the total mortality was higher in patients with arterial hypertension $(13212,21.7 \%$ vs $3774,19.8 \%$, $\mathrm{P}<0.01)$ and patients with diabetes $(3698,22.5 \%$ vs $13288,20.9 \%, \mathrm{P}<0.01)$ compared to those without these disorders. 
The difference on total mortality was particularly marked in patients who had a history of CHF or stroke where having these factors was associated with higher rates of deaths (respectively $8132,28.5 \%$ vs $8854,17.2 \%$ and $2167,31.9 \%$ vs 14819 , $20.3 \%, P<0.01)$.

Table 2

Post-operative mortality of patients who underwent abdominal aortic aneurysm repair according to cardio-metabolic comorbidities

\begin{tabular}{|c|c|c|c|c|c|c|c|c|}
\hline & $\begin{array}{l}\text { Dyslipidemia } \\
N=40989\end{array}$ & $\begin{array}{l}\begin{array}{l}\text { No } \\
\text { dyslipidemia }\end{array} \\
\text { N=38946 }\end{array}$ & $\begin{array}{l}\begin{array}{l}\text { Arterial } \\
\text { hypertension }\end{array} \\
N=60897\end{array}$ & $\begin{array}{l}\text { No arterial } \\
\text { hypertension } \\
N=19038\end{array}$ & $\begin{array}{l}\text { Diabetes } \\
N=16 \\
408\end{array}$ & $\begin{array}{l}\begin{array}{l}\text { No } \\
\text { diabetes }\end{array} \\
N=63 \\
527\end{array}$ & $\begin{array}{l}\text { CHF } \\
N=28 \\
499\end{array}$ & $\begin{array}{l}\text { No } \\
\text { CHF* } \\
\begin{array}{l}\mathrm{N}=51 \\
436\end{array}\end{array}$ \\
\hline $\mathrm{Age}^{\mathrm{y}}$ & $71.0 \pm 9.0$ & $72.1 \pm 10.3$ * & $72.0 \pm 9.4$ & $70.2 \pm 10.6$ * & $\begin{array}{l}70.8 \pm \\
8.8\end{array}$ & $\begin{array}{l}71.8 \pm \\
9.9 *\end{array}$ & $\begin{array}{l}72.9 \pm \\
9.4\end{array}$ & $\begin{array}{l}70.9 \pm \\
9.8 *\end{array}$ \\
\hline $\begin{array}{l}\text { Ruptured } \\
\text { AAA }\end{array}$ & $1485(3.6)$ & $3193(8.2)$ * & $3044(5.0)$ & $1634(8.6)$ * & $\begin{array}{l}690 \\
(4.2)\end{array}$ & $\begin{array}{l}3988 \\
(6.3) \text { * }\end{array}$ & $\begin{array}{l}1361 \\
(4.8)\end{array}$ & $\begin{array}{l}3317 \\
(6.5) \text { * }\end{array}$ \\
\hline $\begin{array}{l}\text { In- } \\
\text { hospital } \\
\text { mortality }\end{array}$ & $1316(3.2)$ & $3265(8.4)$ * & $2803(4.6)$ & $1778(9.3)$ * & $\begin{array}{l}671 \\
(4.1)\end{array}$ & $\begin{array}{l}3910 \\
(6.2) \text { * }\end{array}$ & $\begin{array}{l}1418 \\
(5.0)\end{array}$ & $\begin{array}{l}3163 \\
(6.1) \text { * }\end{array}$ \\
\hline $\begin{array}{l}\text { 1-year } \\
\text { mortality }\end{array}$ & $2586(6.3)$ & $\underset{*}{4} 881(12.5)$ & $5067(8.3)$ & $\underset{\star}{2} 400(12.6)$ & $\begin{array}{l}1342 \\
(8.2)\end{array}$ & $\begin{array}{l}6125 \\
(9.6) \text { * }\end{array}$ & $\begin{array}{l}2855 \\
(10.0)\end{array}$ & $\begin{array}{l}4612 \\
(9.0) \text { * }\end{array}$ \\
\hline \multirow[t]{3}{*}{$\begin{array}{l}\text { Total } \\
\text { mortality }\end{array}$} & 7927 (19.3) & $\begin{array}{l}9059(23.3) \\
*\end{array}$ & $\begin{array}{l}13212 \\
(21.7)\end{array}$ & $\begin{array}{l}3774(19.8) \\
*\end{array}$ & $\begin{array}{l}3698 \\
(22.5)\end{array}$ & $\begin{array}{l}13288 \\
(20.9) *\end{array}$ & $\begin{array}{l}8132 \\
(28.5)\end{array}$ & $\begin{array}{l}8854 \\
(17.2)\end{array}$ \\
\hline & & & SAOS & No SAOS & Smoking & $\begin{array}{l}\text { No } \\
\text { smoking }\end{array}$ & & $\begin{array}{l}\text { No } \\
\text { stroke* }\end{array}$ \\
\hline & $N=17541$ & $N=62394$ & $N=8403$ & $N=71532$ & $\begin{array}{l}N=19 \\
225\end{array}$ & $\begin{array}{l}N=60 \\
710\end{array}$ & $\begin{array}{l}N=6 \\
797\end{array}$ & $\begin{array}{l}N=73 \\
138\end{array}$ \\
\hline $\mathrm{Age}^{\mathrm{y}}$ & $69.5 \pm 9.0$ & $72.2 \pm 9.8$ * & $69.7 \pm 8.6$ & $71.8 \pm 9.8$ * & $\begin{array}{l}66.6 \pm \\
8.7\end{array}$ & $\begin{array}{l}73.2 \pm \\
9.5 *\end{array}$ & $\begin{array}{l}73.2 \pm \\
9.4\end{array}$ & $\begin{array}{l}71.4 \pm \\
9.7 *\end{array}$ \\
\hline $\begin{array}{l}\text { Ruptured } \\
\text { AAA }\end{array}$ & $803(4.6)$ & $3875(6.2)$ * & $298(3.6)$ & $4380(6.1)$ * & $\begin{array}{l}987 \\
(5.2)\end{array}$ & $\begin{array}{l}3691 \\
(6.1) \text { * }\end{array}$ & $\begin{array}{l}313 \\
(4.6)\end{array}$ & $\begin{array}{l}4365 \\
(6.0) \text { * }\end{array}$ \\
\hline $\begin{array}{l}\text { In- } \\
\text { hospital } \\
\text { death }\end{array}$ & $611(3.5)$ & $3970(6.4)$ * & $235(2.8)$ & $4346(6.1)$ * & $\begin{array}{l}719 \\
(3.7)\end{array}$ & $\begin{array}{l}3862 \\
(6.4) \text { * }\end{array}$ & $\begin{array}{l}259 \\
(3.8)\end{array}$ & $\begin{array}{l}4322 \\
(5.9) *\end{array}$ \\
\hline $\begin{array}{l}\text { 1-year } \\
\text { mortality }\end{array}$ & $1148(6.5)$ & $\underset{*}{6} 319(10.1)$ & $500(6.0)$ & $6967(9.7)$ * & $\begin{array}{l}1410 \\
(7.3)\end{array}$ & $\begin{array}{l}6057 \\
(10.0) \text { * }\end{array}$ & $\begin{array}{l}702 \\
(10.3)\end{array}$ & $\begin{array}{l}6765 \\
(9.2) \\
\star \star\end{array}$ \\
\hline $\begin{array}{l}\text { Total } \\
\text { mortality }\end{array}$ & 3349 (19.1) & $\begin{array}{l}13637 \\
(21.9) *\end{array}$ & 1649 (19.6) & $\begin{array}{l}15337 \\
(21.4) *\end{array}$ & $\begin{array}{l}3698 \\
(20.1)\end{array}$ & $\begin{array}{l}13123 \\
(21.6) *\end{array}$ & $\begin{array}{l}2167 \\
(31.9)\end{array}$ & $\begin{array}{l}14819 \\
(20.3) \\
*\end{array}$ \\
\hline \multicolumn{9}{|c|}{ Results are expressed as mean +/- SD or $\mathrm{n}(\%)$. } \\
\hline \multicolumn{9}{|c|}{ CHF: congestive heart failure } \\
\hline \multicolumn{9}{|c|}{ SAOS: Spleeping Apnea Obstructive Syndrome } \\
\hline \multicolumn{9}{|l|}{$\mathrm{y}$ : years } \\
\hline$* p<0.001$ & $p<0.01$ & & & & & & & \\
\hline
\end{tabular}

Factors associated with AAA rupture 
The logistic regression analysis revealed that age over 64 years old and female sex were significantly associated with the risk of AAA rupture (AdjOR = $1.44 \mathrm{Cl} 95 \%=1.34-1.55$ and $\mathrm{AdjOR}=1.15 \mathrm{Cl} 95 \%=1.03-1.27$ ) (Table 3). Chronic kidney disease was also positively associated with the risk of rupture (AdjOR $=1.18 \mathrm{Cl} 95 \%=1.08-1.29)$. On the opposite, cardiometabolic factors, which were less frequent in patients with rupture, were negatively associated with the AAA rupture. The negative association was particularly strong for dyslipidemia and hypertension (AdjOR $=0.44 \mathrm{Cl} 95 \%=0.41-0.47$ and AdjOR $=0.75 \mathrm{CI} 95 \%=0.70-0.81$ respectively) and remained significant for stroke, smoking, SAOS and T2D. A history of chronic respiratory disease was also negatively associated with AAA rupture (AdjOR $=0.76 \mathrm{Cl} 95 \%=0.70-0.82$ ).

Table 3

Factors associated with abdominal aortic aneurysm rupture

\begin{tabular}{|llll|}
\hline \multicolumn{4}{|c|}{ AAA rupture (N= 4 531) } \\
\hline & Adjusted OR & Cl 95\% & P value \\
\hline Age over 64 (ref. <64) & 1.44 & $1.34-1.55$ & $<0.001$ \\
\hline Chronic kidney disease & 1.18 & $1.08-1.29$ & $<0.001$ \\
\hline Women (ref. men) & 1.15 & $1.03-1.27$ & $<0.01$ \\
\hline Dyslipidemia & $\mathbf{0 . 4 4}$ & $0.41-0.47$ & $<0.001$ \\
\hline Hypertension & $\mathbf{0 . 7 5}$ & $0.70-0.81$ & $<0.001$ \\
\hline Chronic respiratory disease & $\mathbf{0 . 7 6}$ & $0.70-0.82$ & $<0.001$ \\
\hline Stroke & $\mathbf{0 . 8 0}$ & $0.72-0.88$ & $<0.001$ \\
\hline Smoking & $\mathbf{0 . 8 4}$ & $0.77-0.91$ & 0.007 \\
\hline SAOS & $\mathbf{0 . 8 4}$ & $0.73-0.95$ & $<0.01$ \\
\hline T2D & $\mathbf{0 . 8 6}$ & $0.78-0.94$ & 0.001 \\
\hline AAA: abdominal aortic aneurysm & & \\
\hline CI95\%: Confidence Limits 95\% & & \\
\hline OR: Odds ratio & & & \\
\hline ref.: Reference & & & \\
\hline SAOS: Sleeping Apnea Obstructive Syndrome & & \\
\hline T2D: type 2 diabetes & & & \\
\hline
\end{tabular}

\section{Factors associated with post-operative mortality after AAA repair}

In total, 61146 patients (76.5\%) had at least one follow-up after the release date from the first AAA repair and were included in the analysis (Fig. 1). The analysis excluded in-hospital mortality during the first AAA repair. The mean follow-up was 3.5 years +/- 2.5. The total number of deaths was 12400 (20.3\%), among them 3817 after open repair and 8583 after EVAR. Age over 64 years old and the presence of AAA rupture were positively associated with the risk of post-operative total mortality (AdjHR = $1.59 \mathrm{Cl} 95 \%=1.51-1.67$ and AdjHR = $1.49 \mathrm{Cl} 95 \%=1.36-1.62)$ (Table 4). In-hospital readmission at 30-day was a strong predictor of post-operative mortality ( $\mathrm{AdjHR}=1.92, \mathrm{Cl} 95 \%=1.84-2.00)$. The association was particularly strong for open repair where the risk of mortality was doubled in case of a re-hospitalization within 30-days following AAA repair (AdjHR = 2.03, Cl95\% 1.88-2.19). Compared to open repair, EVAR was associated with increased risk of mortality (AdjHR $=1.48, \mathrm{Cl} 95 \%=1.42-1.54)$. Among cardiovascular diseases, CHF and history of stroke were significantly associated with the risk of post-operative mortality (Adj HR. $=1.40, \mathrm{Cl} 95 \%=1.35-1.46$ and $\mathrm{AdjHR}=1.19, \mathrm{Cl} 95 \%=1.14-1.23$, 
respectively). Other comorbidities such as chronic respiratory disease or chronic kidney disease were also predictive of mortality. Among cardiovascular risk factors, only T1D and smoking were positively associated with post-operative mortality. On the opposite, dyslipidemia, obesity and SAOS, were negatively associated with post-operative mortality. 
Risk factors of mortality after abdominal aortic aneurysm surgical repair

\begin{tabular}{|c|c|c|c|c|c|c|}
\hline & $\begin{array}{l}\text { Total* } \\
\mathrm{N}=61146\end{array}$ & & $\begin{array}{l}\text { Open repair* } \\
N=22250\end{array}$ & & $\begin{array}{l}\text { EVAR* } \\
N=38896\end{array}$ & \\
\hline & $\begin{array}{l}\text { Adjusted Hazard } \\
\text { Ratio }\end{array}$ & Cl 95\% & $\begin{array}{l}\text { Adjusted Hazard } \\
\text { Ratio }\end{array}$ & Cl 95\% & $\begin{array}{l}\text { Adjusted Hazard } \\
\text { Ratio }\end{array}$ & Cl $95 \%$ \\
\hline $\begin{array}{l}\text { In-hospital readmission at } \\
30 \text { days }\end{array}$ & 1.92 & $\begin{array}{l}1.84- \\
2.00\end{array}$ & 2.03 & $\begin{array}{l}1.88- \\
2.19\end{array}$ & 1.88 & $\begin{array}{l}1.79- \\
1.98\end{array}$ \\
\hline Age $>64$ years & 1.59 & $\begin{array}{l}1.51- \\
1.67\end{array}$ & 1.53 & $\begin{array}{l}1.42- \\
1.65\end{array}$ & 1.63 & $\begin{array}{l}1.52- \\
1.74\end{array}$ \\
\hline Ruptured AAA & 1.49 & $\begin{array}{l}1.36- \\
1.62\end{array}$ & 1.48 & $\begin{array}{l}1.34- \\
1.63\end{array}$ & 1.60 & $\begin{array}{l}1.26- \\
2.02\end{array}$ \\
\hline EVAR (ref. open repair) & 1.48 & $\begin{array}{l}1.42- \\
1.54\end{array}$ & & & & \\
\hline CHF & 1.40 & $\begin{array}{l}1.35- \\
1.46\end{array}$ & 1.33 & $\begin{array}{l}1.24- \\
1.42\end{array}$ & 1.44 & $\begin{array}{l}1.38- \\
1.51\end{array}$ \\
\hline $\begin{array}{l}\text { Chronic respiratory } \\
\text { disease }\end{array}$ & 1.33 & $\begin{array}{l}1.28- \\
1.38\end{array}$ & 1.35 & $\begin{array}{l}1.26- \\
1.45\end{array}$ & 1.32 & $\begin{array}{l}1.26- \\
1.38\end{array}$ \\
\hline Chronic kidney disease & 1.31 & $\begin{array}{l}1.26- \\
1.36\end{array}$ & 1.30 & $\begin{array}{l}1.21- \\
1.39\end{array}$ & 1.31 & $\begin{array}{l}1.25- \\
1.37\end{array}$ \\
\hline T1D & 1.30 & $\begin{array}{l}1.20- \\
1.40\end{array}$ & 1.33 & $\begin{array}{l}1.16- \\
1.52\end{array}$ & 1.28 & $\begin{array}{l}1.17- \\
1.41\end{array}$ \\
\hline Stroke & 1.19 & $\begin{array}{l}1.14- \\
1.23\end{array}$ & 1.21 & $\begin{array}{l}1.12- \\
1.30\end{array}$ & 1.18 & $\begin{array}{l}1.12- \\
1.23\end{array}$ \\
\hline Female sex & 1.06 & $\begin{array}{l}1.00- \\
1.13\end{array}$ & 1.15 & $\begin{array}{l}1.03- \\
1.28\end{array}$ & & \\
\hline Smoking & 1.06 & $\begin{array}{l}1.02- \\
1.11\end{array}$ & 1.13 & $\begin{array}{l}1.06- \\
1.22\end{array}$ & & \\
\hline Dyslipidemia & 0.72 & $\begin{array}{l}0.69- \\
0.75\end{array}$ & 0.75 & $\begin{array}{l}0.71- \\
0.81\end{array}$ & 0.71 & $\begin{array}{l}0.68- \\
0.74\end{array}$ \\
\hline Obesity & 0.83 & $\begin{array}{l}0.79- \\
0.86\end{array}$ & 0.79 & $\begin{array}{l}0.71- \\
0.89\end{array}$ & 0.84 & $\begin{array}{l}0.80- \\
0.89\end{array}$ \\
\hline SAOS & 0.83 & $\begin{array}{l}0.79- \\
0.86\end{array}$ & 0.81 & $\begin{array}{l}0.74- \\
0.88\end{array}$ & 0.85 & $\begin{array}{l}0.79- \\
0.91\end{array}$ \\
\hline
\end{tabular}

* Population of patients who had at least one follow-up i.e admission at the hospital after the AAA surgical repair Results are expressed as mean $+/$ - SD or $\mathrm{n}(\%)$.

AAA: abdominal aortic aneurysm

CHF: congestive heart failure

EVAR: endovascular aneurysm repair

SAOS: Sleeping Apnea Obstructive Syndrome

T2D: type 2 diabetes

T1D: type 1 diabetes 


\begin{tabular}{|c|c|c|c|c|c|c|}
\hline \multirow{3}{*}{ Arterial hypertension } & \multirow{2}{*}{$\begin{array}{l}\text { Total* } \\
\mathrm{N}=61146\end{array}$} & & \multirow{2}{*}{\multicolumn{2}{|c|}{$\begin{array}{l}\text { Open repair* } \\
N=22250\end{array}$}} & \multirow{2}{*}{\multicolumn{2}{|c|}{$\begin{array}{l}\text { EVAR* } \\
N=38896\end{array}$}} \\
\hline & & & & & & \\
\hline & 0.96 & $\begin{array}{l}0.92- \\
1.01\end{array}$ & 0.93 & $\begin{array}{l}0.85- \\
1.02\end{array}$ & 0.97 & $\begin{array}{l}0.92- \\
1.03\end{array}$ \\
\hline T2D & 0.96 & $\begin{array}{l}0.92- \\
1.01\end{array}$ & 1.00 & $\begin{array}{l}0.93- \\
1.09\end{array}$ & 0.95 & $\begin{array}{l}0.90- \\
1.00\end{array}$ \\
\hline \multicolumn{7}{|c|}{ * Population of patients who had at least one follow-up i.e admission at the hospital after the AAA surgical repair } \\
\hline \multicolumn{7}{|c|}{ Results are expressed as mean $+/-$ SD or $n(\%)$. } \\
\hline \multicolumn{7}{|c|}{ AAA: abdominal aortic aneurysm } \\
\hline \multicolumn{7}{|c|}{ CHF: congestive heart failure } \\
\hline \multicolumn{7}{|c|}{ EVAR: endovascular aneurysm repair } \\
\hline \multicolumn{7}{|c|}{ SAOS: Sleeping Apnea Obstructive Syndrome } \\
\hline \multicolumn{7}{|l|}{ T2D: type 2 diabetes } \\
\hline T1D: type 1 diabetes & & & & & & \\
\hline
\end{tabular}

\section{Discussion}

This ten-year observational nationwide cohort of 79935 patients reports for the first time the outcomes of patients from the French National Health data and represents one of the largest European retrospective study on AAA repair ${ }^{6,8,9}$.

The proportion of patients who had EVAR/ open repair was balanced (approximatively 0.6) and was similar to the practice observed in other European countries ${ }^{6,8}$. Ruptured AAA were most often treated using open repair ( $96.2 \%$ of cases). A population-based study in Finland between 2000 and 2014 revealed similar results, with 1627 treated with open repair over the 1687 ruptured AAA (96.4\%). Several observational and multicenter randomized clinical trials have demonstrated improved outcomes after EVAR for ruptured AAA 3,12 and national trends in the United States confirmed that EVAR is increasingly used in the treatment of AAA rupture ${ }^{13,14}$. Changes in the management of ruptured AAA are thus to be expected in France within the next decade.

The in-hospital mortality of patients operated for a non-ruptured AAA in France was 3.6\%. A nationwide analysis of 84631 patients in Germany reported comparable rate, with $3.3 \%$ of in-hospital mortality in patients operated for intact $A A A^{8}$. In case of aortic rupture, the in-hospital mortality following AAA repair was high (40\%) and was concordant with other published studies ${ }^{15,16}$. Age over 64 years old and female sex were significantly associated with the risk of AAA rupture, which is concordant with the current literature 5,17 .

Interestingly, we found that the prevalence of cardiovascular comorbidities and metabolic disorders was significantly lower in patients with ruptured AAA compared to non-ruptured AAA. As a consequence, the odd ratio analysis showed that cardiometabolic factors, were negatively associated with the risk of AAA rupture. The paradoxical negative association observed in our study could be explained by a delay in the diagnosis of AAA in patients who did not have cardio-metabolic comorbidities. It can be hypothesized that patients with comorbidities benefit from a closer follow-up and a screening of associated cardiovascular diseases. AAA in these patients may be discovered at earlier, asymptomatic and non-ruptured stage. On the other hand, it can be assumed that most of the patients who presented a rupture may have not been previously diagnosed for an AAA and may have not benefited from a systematic screening of cardiovascular comorbidities. 
It is thus possible that cardio-metabolic comorbidities may be underdiagnosed and underestimated in patients admitted for a ruptured $A A A$.

Our results underline the interest of population screening for AAA. Randomized clinical trials have demonstrated that ultrasound screening was effective to reduce AAA-related mortality, even in case of low prevalence of AAA ${ }^{18-21}$. The review published by the National Institute for Health and Care Excellence (UK) regarding risk factors for predicting presence of an abdominal aortic aneurysm showed strong evidence that the risk of AAA increased with age ${ }^{22}$. Although there was low or moderate quality evidence for cardiovascular diseases, hypertension and dyslipidemia, the committee agreed that taking into account risk factors could improve detection rates for opportunistic screening of AAA.

In case of AAA rupture, the death occurred more frequently during the hospital stay of the first AAA repair (1 873 over 2425 , $77.2 \%)$. Thus, it is not surprising that the in-hospital mortality was significantly lower in patients with cardio-metabolic comorbidities compared to those who did not, given the fact that they had a lower proportion of ruptured AAA. The same trend was observed for the 1-year mortality. As recommended by the Society of Vascular Surgery, patients with known cardio-metabolic comorbities benefit from a thorough pre-operative evaluation and a close follow-up, which could partly contribute to limit the mortality ${ }^{3}$.

However, our results on total long-term mortality rather suggested a worse prognosis in patients with cardio-metabolic comorbidities. Other studies have also identified heart failure, ischemic heart disease, cerebrovascular disease and diabetes as risk factors of mortality following elective AAA repair ${ }^{23,24}$. Nevertheless, we found in our cohort that the total mortality was significantly lower in patients presenting dyslipidemia, obesity, SAOS or smoking compared to those who did not have these comorbidities. Note that non-smokers were significantly older than smokers and could explain this result. Johal et al. recently aimed to examine patterns of 10-year survival after elective repair of unruptured AAA and investigated the survival among patients of different age and different co-morbidity score profiles from the English National Health register ${ }^{25}$. The long-term survival patterns after elective open repair and EVAR for unruptured AAA varied markedly across patients with different age and co-morbidity profiles. This underlines the complex impact of comorbidities on the postoperative outcomes and suggests that multiparametric predictive scores would be of interest.

The survival analysis after discharge from the first AAA repair revealed that aging, the presence of an aortic rupture, inhospital readmission at 30 days, chronic respiratory and kidney diseases were predictive factors of post-operative mortality. These results are concordant with a meta-analysis including 45 studies investigating factors influencing survival following AAA repair ${ }^{26}$. End stage renal disease, chronic obstructive pulmonary disease, age, cardiac failure, cerebrovascular diseases were associated with poor long-term survival. In our cohort, we found that in-hospital mortality was higher in patients who had open repair, and could be partially explained by the higher proportion of ruptured AAA. However, EVAR was associated with increased risk of long-term mortality after discharge from the hospital. Randomized clinical trials have suggested that EVAR was associated with early survival gain, while open repair showed similar or late survival benefits ${ }^{27-30}$.

\section{Limitations}

This a retrospective observational study based on electronic administrative database and the results may depend on the coding system. Nevertheless, the French National insurance information system uses codes based on standardized definitions. Data are extracted by medical doctors and certified reviewers and the quality of the French National electronic health data is audited annually by experts. The medical treatments of patients were not available and the deaths occurring in the absence of hospitalization could not be recorded in the database. However, given the age of the population and the total mortality rate $(21.3 \%)$, it can be assumed that most of the patients presenting a severe and potentially life-threatening disease may have been hospitalized. Although the results could not be classified according to the severity of the comorbidities, the size of the cohort allowed adequate statistical power to investigate the differences among the groups. 


\section{Conclusion}

This ten-year observational nationwide study investigating the outcomes of patients who underwent AAA repair in France between 2010-2019 revealed a total long-term mortality of $21.3 \%$. Open repair was associated with a higher prevalence of AAA rupture and in-hospital mortality, while EVAR was associated with a higher risk of mortality after hospital discharge. The cardio-metabolic comorbidities were significantly lower in patients with AAA rupture compared to patients with intact AAA. Our results suggest that AAA may be underdiagnosed in patients without known comorbidities and that cardiovascular comorbidities may be underestimated in patients with ruptured AAA. This study sheds light on the importance to diagnose associated cardiovascular comorbidities in patients undergoing AAA repair. It also highlights the interest of screening for AAA and should encourage institutions to perform it to reduce related mortality.

\section{Declarations}

\section{Acknowledgements: none}

\section{Author contributions:}

JR, FL and LB wrote the main manuscript. RF and LB collected the data. JR, FL, RF, ZM, CP and LB authors interpreted and analyzed the data. JR, FL, RF and LB prepared the figures. JR, FL, RF, ZM, CP and LB critically reviewed the manuscript.

\section{Additional Information:}

- Sources of Funding: none

- Disclosures: The authors declare no conflict of interest.

- Ethical approval for research: The study was conducted in accordance with the French National Health Data Institute regulation.

\section{References}

1. Wanhainen, A. et al. (eds) Editor's Choice - European Society for Vascular Surgery (ESVS) 2019 Clinical Practice Guidelines on the Management of Abdominal Aorto-iliac Artery AneurysmsEur J Vasc Endovasc Surg 57, 8-93, doi:10.1016/j.ejvs.2018.09.020 (2019).

2. Sakalihasan, N., Limet, R. \& Defawe, O. D. Abdominal aortic aneurysm. Lancet.365, 1577-1589 https://doi.org/10.1016/S0140-6736(05)66459-8 (2005).

3. Chaikof, E. L. et al. The Society for Vascular Surgery practice guidelines on the care of patients with an abdominal aortic aneurysm. J Vasc Surg 67, 2-77 e72, doi:10.1016/j.jvs.2017.10.044 (2018).

4. Dua, A. et al. Unplanned 30-day readmissions after endovascular aneurysm repair: An analysis using the Nationwide Readmissions Database. J Vasc Surg.70, 1603-1611 https://doi.org/10.1016/j.jvs.2019.02.034 (2019).

5. Nordon, I. M., Hinchliffe, R. J., Loftus, I. M. \& Thompson, M. M. Pathophysiology and epidemiology of abdominal aortic aneurysms. Nat Rev Cardiol.8, 92-102 https://doi.org/10.1038/nrcardio.2010.180 (2011).

6. Laine, M. T. et al. A Population-Based Study of Abdominal Aortic Aneurysm Treatment in Finland 2000 to 2014. Circulation.136, 1726-1734 https://doi.org/10.1161/CIRCULATIONAHA.117.028259 (2017).

7. Piepoli, M. F. et al. 2016 European Guidelines on cardiovascular disease prevention in clinical practice: The Sixth Joint Task Force of the European Society of Cardiology and Other Societies on Cardiovascular Disease Prevention in Clinical Practice (constituted by representatives of 10 societies and by invited experts)Developed with the special contribution of the European Association for Cardiovascular Prevention \& Rehabilitation (EACPR). Eur Heart J 37, 2315-2381, doi:10.1093/eurheartj/ehw106 (2016). 
8. Trenner, M., Kuehnl, A., Reutersberg, B., Salvermoser, M. \& Eckstein, H. H. Nationwide analysis of risk factors for inhospital mortality in patients undergoing abdominal aortic aneurysm repair. Br J Surg.105, 379-387 https://doi.org/10.1002/bjs.10714 (2018).

9. Karthikesalingam, A. et al. The impact of endovascular aneurysm repair on mortality for elective abdominal aortic aneurysm repair in England and the United States. J Vasc Surg 64, 321-327 e322, doi:10.1016/j.jvs.2016.01.057 (2016).

10. Bailly, L., Fabre, R., Pradier, C. \& lannelli, A. Colorectal Cancer Risk Following Bariatric Surgery in a Nationwide Study of French Individuals With Obesity. JAMA Surg.https://doi.org/10.1001/jamasurg.2020.0089 (2020).

11. Thereaux, J. et al. Long-term follow-up after bariatric surgery in a national cohort. Br J Surg.104, 1362-1371 https://doi.org/10.1002/bjs.10557 (2017).

12. Investigators, I. T. et al. Endovascular or open repair strategy for ruptured abdominal aortic aneurysm: 30 day outcomes from IMPROVE randomised trial. BMJ.348, f7661 https://doi.org/10.1136/bmj.f7661 (2014).

13. Lesperance, K., Andersen, C., Singh, N., Starnes, B. \& Martin, M. J. Expanding use of emergency endovascular repair for ruptured abdominal aortic aneurysms: disparities in outcomes from a nationwide perspective. J Vasc Surg.47, 11651170 discussion 1170 - 1161 https://doi.org/10.1016/j.jvs.2008.01.055 (2008).

14. Mureebe, L. et al. National trends in the repair of ruptured abdominal aortic aneurysms. J Vasc Surg.48, 1101-1107 https://doi.org/10.1016/j.jvs.2008.06.031 (2008).

15. Hoornweg, L. L. et al. Meta analysis on mortality of ruptured abdominal aortic aneurysms. Eur J Vasc Endovasc Surg.35, 558-570 https://doi.org/10.1016/j.ejvs.2007.11.019 (2008).

16. Visser, P., Akkersdijk, G. J. \& Blankensteijn, J. D. In-hospital operative mortality of ruptured abdominal aortic aneurysm: a population-based analysis of 5593 patients in The Netherlands over a 10-year period. Eur J Vasc Endovasc Surg.30, 359-364 https://doi.org/10.1016/j.ejvs.2005.05.005 (2005).

17. Moll, F. L. et al. Management of abdominal aortic aneurysms clinical practice guidelines of the European society for vascular surgery. Eur J Vasc Endovasc Surg.41 (Suppl 1), S1-S58 https://doi.org/10.1016/j.ejvs.2010.09.011 (2011).

18. Ashton, H. A. et al. The Multicentre Aneurysm Screening Study (MASS) into the effect of abdominal aortic aneurysm screening on mortality in men: a randomised controlled trial. Lancet.360, 1531-1539 https://doi.org/10.1016/s01406736(02)11522-4 (2002).

19. Spronk, S. et al. Cost-effectiveness of screening for abdominal aortic aneurysm in the Netherlands and Norway. Br J Surg.98, 1546-1555 https://doi.org/10.1002/bjs.7620 (2011).

20. Glover, M. J., Kim, L. G., Sweeting, M. J., Thompson, S. G. \& Buxton, M. J. Cost-effectiveness of the National Health Service Abdominal Aortic Aneurysm Screening Programme in England. Br J Surg.101, 976-982 https://doi.org/10.1002/bjs.9528 (2014).

21. Svensjo, S., Mani, K., Bjorck, M., Lundkvist, J. \& Wanhainen, A. Screening for abdominal aortic aneurysm in 65-year-old men remains cost-effective with contemporary epidemiology and management. Eur J Vasc Endovasc Surg.47, 357365 https://doi.org/10.1016/j.ejvs.2013.12.023 (2014).

22. in Risk factors for predicting presence of an abdominal aortic aneurysm: Abdominal aortic aneurysm: diagnosis and management: Evidence review A (2020).

23. Marques-Rios, G., Oliveira-Pinto, J. \& Mansilha, A. Predictors of long-term mortality following elective endovascular repair of abdominal aortic aneurysms. Int Angio/.37, 277-285 https://doi.org/10.23736/S0392-9590.18.03988-3 (2018).

24. Schlosser, F. J. et al. Mortality after elective abdominal aortic aneurysm repair. Ann Surg.251, 158-164 https://doi.org/10.1097/SLA.0b013e3181bc9c4d (2010).

25. Johal, A. S. et al. Long-term survival after endovascular and open repair of unruptured abdominal aortic aneurysm. $B r$ J Surg.106, 1784-1793 https://doi.org/10.1002/bjs.11215 (2019).

Page $14 / 16$ 
26. Khashram, M., Williman, J. A., Hider, P. N., Jones, G. T. \& Roake, J. A. Systematic Review and Meta-analysis of Factors Influencing Survival Following Abdominal Aortic Aneurysm Repair. Eur J Vasc Endovasc Surg.51, 203-215 https://doi.org/10.1016/j.ejvs.2015.09.007 (2016).

27. Blankensteijn, J. D. et al. Two-year outcomes after conventional or endovascular repair of abdominal aortic aneurysms. N Engl J Med.352, 2398-2405 https://doi.org/10.1056/NEJMoa051255 (2005).

28. Greenhalgh, R. M. et al. Comparison of endovascular aneurysm repair with open repair in patients with abdominal aortic aneurysm (EVAR trial 1), 30-day operative mortality results: randomised controlled trial. Lancet.364, 843-848 https://doi.org/10.1016/S0140-6736(04)16979-1 (2004).

29. Becquemin, J. P. et al. A randomized controlled trial of endovascular aneurysm repair versus open surgery for abdominal aortic aneurysms in low- to moderate-risk patients. J Vasc Surg.53 (e1161), 1167-1173 https://doi.org/10.1016/j.jvs.2010.10.124 (2011).

30. Patel, R., Sweeting, M. J., Powell, J. T. \& Greenhalgh, R. M. \& investigators, E. t. Endovascular versus open repair of abdominal aortic aneurysm in 15-years' follow-up of the UK endovascular aneurysm repair trial 1 (EVAR trial 1): a randomised controlled trial. Lancet 388, 2366-2374, doi:10.1016/S0140-6736(16)31135-7 (2016).

\section{Figures}

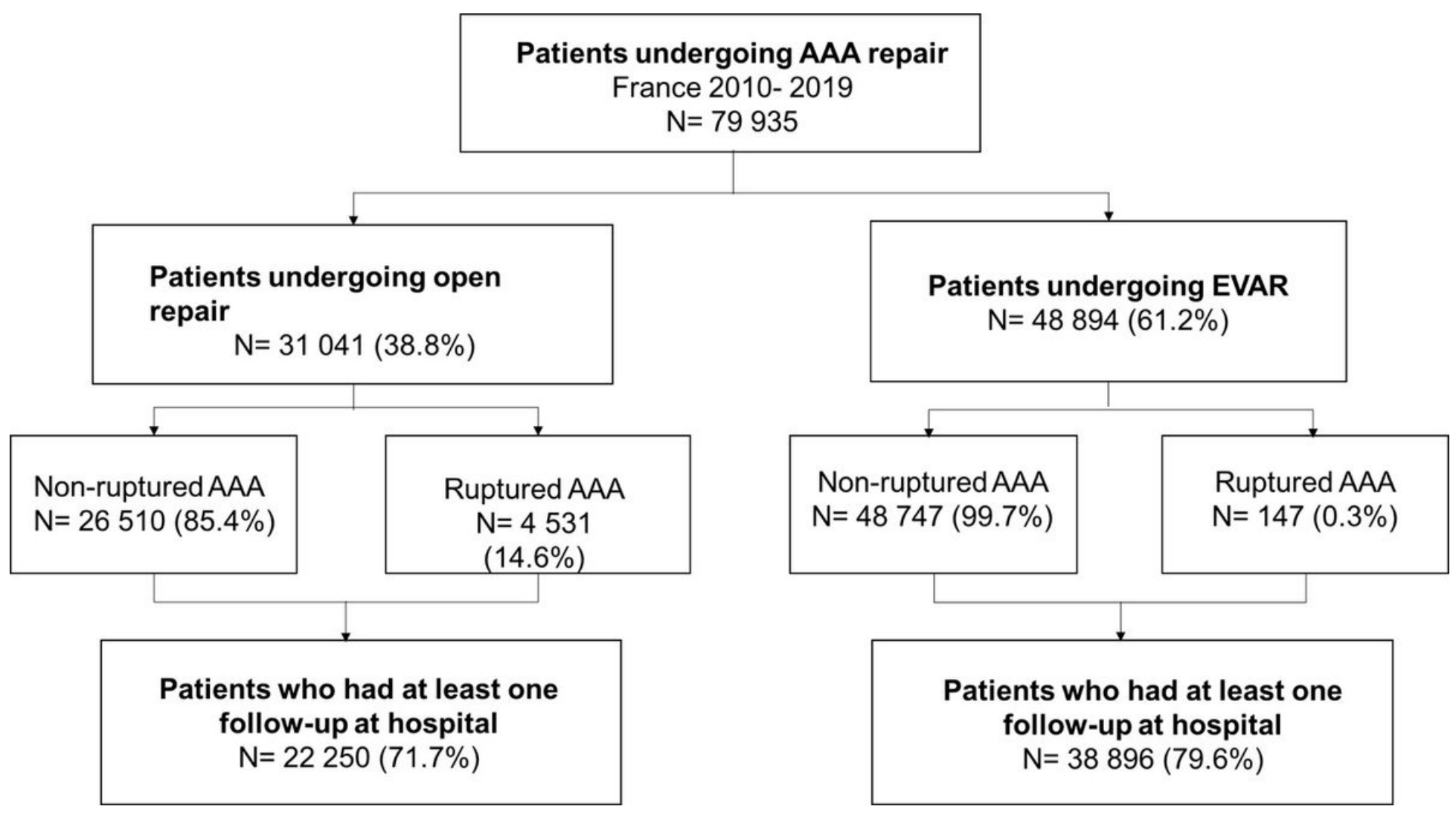

Figure 1

Flow chart of the study population Data are expressed as $n(\%)$.

\section{Supplementary Files}

This is a list of supplementary files associated with this preprint. Click to download. 
- Supplementarydata.docx

Page 16/16 